\title{
Algoritmos para geração de padrões aplicado ao problema de corte unidimensional
}

\author{
RESUMO
}

\section{Gerson Ulbricht}

gerson.ulbricht@ifsc.edu.br

Instituto Federal de Santa Catarina (IFSC), Jaraguá do Sul, SC, Brasil

\section{Cristian de Souza}

cristiandsouza@hotmail.com

Instituto Federal de Santa Catarina

(IFSC), Jaraguá do Sul, SC, Brasil
Este trabalho apresenta um estudo sobre um problema de cortes unidimensional a partir do qual foi desenvolvido um algoritmo para auxílio na geração de padrões de cortes. A partir dos padrões gerados com o algoritmo e após verificações em trabalhos da literatura, foi proposto um modelo de otimização envolvendo um problema de programação linear cuja resolução foi implementada com auxílio do suplemento Solver, disponível no software Microsoft Excel. O algoritmo desenvolvido para geração dos padrões foi implementado em linguagem Scilab, por se tratar de um software de licença livre. 0 modelo de otimização foi inspirado em um problema existente numa indústria que trabalha com corte de eletrodutos, porém tomou-se o cuidado de generalizá-lo para que possa ser utilizado em outras aplicações envolvendo cortes unidimensionais. Resultados apontaram que o modelo desenvolvido é uma boa ferramenta no auxílio à decisão que abre possibilidade para a busca da redução de desperdícios envolvendo matéria prima, indicando uma área promissora para desenvolvimento computacional e pesquisas futuras.

PALAVRAS-CHAVE: Algoritmo. Problema de Cortes. Otimização. 


\section{INTRODUÇÃO}

No mercado atual, as empresas precisam aprimorar-se buscando reduzir custos de produção dentro da sua gama de mercado. Uma das premissas fundamentais para o bom desempenho financeiro é a correta compra da matéria prima evitando assim desperdícios ou ainda estoques imobilizados, o que pode gerar custos com estocagem, gastos com espaço físico e ainda depreciação.

Problemas que tratam de cortes de materiais, de modo geral, são frequentes em grande parte das indústrias. Pode-se imaginar que a operação de corte é uma simples tarefa (ato de cortar peças), porém atentando-se ao fato de se cumprir demandas pré-estabelecidas para cada tipo de medida, nota-se que grandes desperdícios podem ser gerados, se não houver um plano adequado para realização da tarefa de cortes.

Em grande parte das operações que envolvem cortes de materiais há perdas significativas ocasionadas pela sobra de materiais não utilizáveis bem como pelo próprio processo de corte ocasionado pelo desperdício da lâmina da serra. Mesmo que esse material desperdiçado possa ser reciclado, o procedimento de reciclagem em si, gera custos financeiros e ambientais, pois muitas vezes é necessário novo processo de fundição, prensagem entre outros.

A busca pela redução de desperdícios deve ser constantemente observada nas indústrias (BUENO, 2007). Nas tarefas de corte, o objetivo de reduzir desperdícios está diretamente ligado ao planejamento de tarefas, de modo a estabelecer um bom arranjo dos padrões de corte, ou seja, a quantidade e tamanho dos fragmentos a serem cortados em cada peça de tamanho padrão. Em muitos casos não há qualquer planejamento anterior ao processo de corte, sendo esse realizado de forma arbitrária, sem utilização de quaisquer métodos científicos (SILVEIRA et al, 2004).

Neste trabalho, baseado em um problema envolvendo cortes de eletrodutos, foi proposto um algoritmo computacional capaz de encontrar os padrões de cortes válidos, que alimentam o modelo matemático de otimização. Os códigos foram desenvolvidos na linguagem Scilab, por se tratar de uma linguagem de licença livre (o software Scilab pode ser obtido gratuitamente em www.scilab.org), o que possibilita a redução de custos financeiros para implantação industrial de um sistema de auxílio ao planejamento do processo de cortes.

\section{FUNDAMENTAÇÃO}

O desenvolvimento deste trabalho deu-se a partir de demanda surgida em uma empresa situada no município de Jaraguá do Sul - SC. Essa empresa fabrica geradores elétricos, sendo que para o cabeamento necessita de grandes quantidades de eletrodutos rígidos de diversas medidas, os quais tem por objetivo a proteção dos cabos, evitando exposição aos operadores e também a organização do cabeamento. Simone (2000, p.34), Borges Neto e Carvalho (2013, p. 33) e Walenia (2010, p. 12) apresentam maiores detalhes sobre essa aplicação.

A Figura 1 mostra detalhes da utilização de eletrodutos rígidos em montagem de painéis elétricos. 
Figura 1 - Exemplo de encaminhamento de cabos até painéis

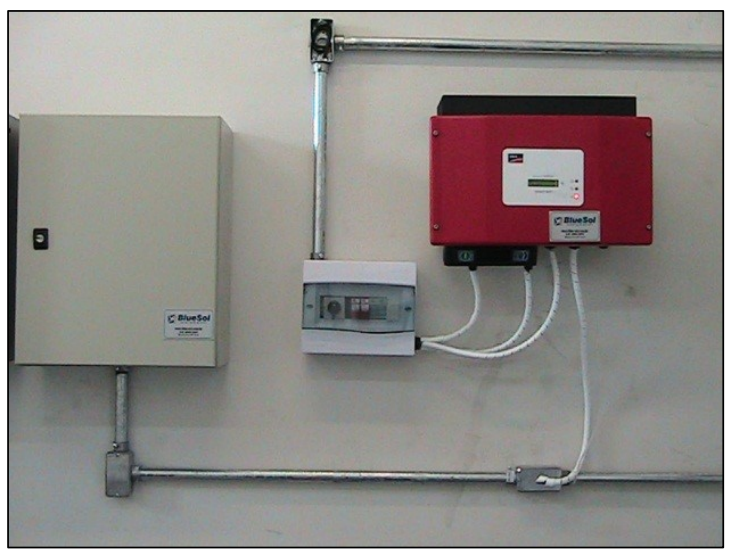

Fonte: http://www.blue-sol.com. Acesso em 15/10/2019.

Ao executar um projeto a empresa objeto de estudo deste artigo, necessita fazer um pedido de uma quantidade fixa de eletrodutos. Esses eletrodutos são fornecidos numa medida única, de $3 \mathrm{~m}(3000 \mathrm{~mm})$ e deverão ser posteriormente seccionados de modo a atender as demandas do projeto.

O sistema de corte nessa empresa é feito manualmente sem qualquer planejamento prévio, o que gera sobra de matérias, ou seja, pequenos fragmentos que não têm utilização no projeto, e que geram resíduos.

A correta aplicação de um plano de cortes contribui para a redução de desperdícios, tendo impacto ambiental e financeiro quando aplicado a uma situação real (ANDRADE, 2000).

Existem ferramentas matemáticas já desenvolvidas, voltadas ao auxílio para a tomada de decisões aplicadas a problemas de cortes (MOURA et. al, 2019), (GOLDBARG e LUNA, 2005). O problema é que, quando há demandas por vários tamanhos diferentes de peças, os problemas vão ganhando mais variáveis e ficando cada vez mais complexos de serem resolvidos (ARENALES et al., 2015).

Um modelo matemático envolvendo programação linear foi apresentado por Wavrzynczak (2015). Neste caso, o autor selecionou os possíveis padrões de cortes, chamados de padrões de cortes válidos, por meio de um processo de inspeção manual, com auxílio de uma planilha de cálculo.

Sendo assim, é ideia principal é que haja a verificação por meio do projeto, das quantidades de cada medida que devem ser utilizadas, de modo que essas quantidades sejam cortadas de modo planejado.

A partir das demandas estabelecidas, que são as quantidades a serem cortadas de cada medida a ser utilizada na linha de produção, pode ser estabelecido um plano de cortes (OLIVEIRA et al, 2013). Deve-se lembrar que em um processo de cortes são geradas as medidas pré-estipuladas, porém há 2 tipos de desperdício, sendo um deles gerado pela sobra de material e outro pela largura da serra conforme mostra a Figura 2. Maiores detalhes sobre esses dois tipos de desperdício podem ser vistos em Wavrzynczak (2015). 
Figura 2 - Exemplo genérico de padrão de corte.

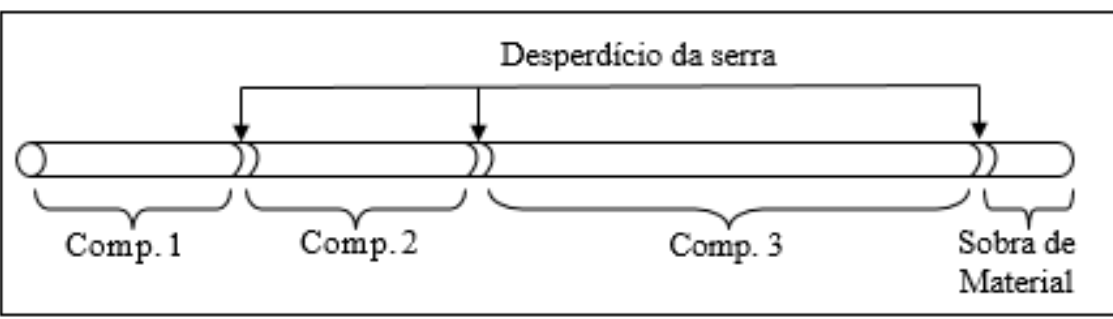

Fonte: Autoria própria (2020).

\section{METODOLOGIA}

\section{PADRÕES DE CORTES}

Conforme mostrado na Tabela 1, existem no problema aqui estudado, o rol de 13 diferentes medidas, as quais foram projetadas pelo departamento de projetos específicos. Sendo assim, o eletroduto de tamanho padrão ( $3 \mathrm{~m}$ ou seja, $3000 \mathrm{~mm}$ ) deverá ser cortado obedecendo a medida projetada, o que pode gerar desperdício de material gerado pelas extremidades que não serão utilizadas e também desperdício de cavaco referente ao corte da serra. Foi considerado neste trabalho, a largura da serra de $2,5 \mathrm{~mm}$, que é a medida atualmente utilizada na empresa onde foi realizado o estudo de caso.

A Tabela 1, mostra as quantidades a serem utilizadas em um pedido da empresa, tomado como exemplo.

Tabela 1 - Medidas e quantidades de peças

\begin{tabular}{ccc} 
Descrição & Medida $(\mathrm{mm})$ & Quantidade \\
Comprimento 1 & 254,5 & 60 \\
Comprimento 2 & 1962 & 30 \\
Comprimento 3 & 1132 & 10 \\
Comprimento 5 & 1466 & 20 \\
Comprimento 6 & 100 & 10 \\
Comprimento 7 & 404 & 30 \\
Comprimento 8 & 1000 & 180 \\
Comprimento 9 & 1500 & 40 \\
Comprimento 10 & 1454,93 & 10 \\
Comprimento 11 & 736 & 20 \\
Comprimento 12 & 703,75 & 40 \\
Comprimento 13 & 514,6 & 10 \\
\hline
\end{tabular}

Fonte: Autoria própria (2019)

Considerando as medidas estipuladas para corte, conforme mostrado na Tabela 1 nota-se que é possível encontrar uma grande quantidade de diferentes padrões de corte gerando assim, diferentes desperdícios para cada padrão. 
Sendo assim, é uma árdua tarefa encontrar os padrões de corte válidos que são combinações de medidas possíveis de serem cortadas em uma barra padrão, gerando ou não, um desperdício. Sendo assim, quanto maior a quantidade de padrões de corte disponíveis, melhor a gama de possibilidades de escolha de quais padrões serão utilizados de modo a atender as demandas de cada medida com o menor desperdício possível (LOESCH e HEIN, 2009).

Buscando gerar os padrões de corte que serão utilizados na elaboração do modelo matemático, foi gerado um algoritmo, o qual analisa os padrões gerados e retorna somente os padrões válidos (AGUILAR, 2008), (FORBELLONE, 2005).

Entende-se por padrão válido, um padrão que possibilita o corte de uma ou mais medidas programadas, gerando consequentemente um desperdício de corte de serra e cujo desperdício de matéria prima (ponteira restante do processo de corte), seja maior ou igual a zero e menor que a menor das medidas a serem utilizadas.

\section{ALGORITMOS PROPOSTOS PARA GERAÇÃO DE PADRÕES DE CORTE}

Nos tópicos a seguir são apresentados 2 algoritmos para geração de padrões. O primeiro deles baseado na busca exaustiva e o segundo na busca aleatória de combinações.

\section{Algoritmo de Busca Exaustiva}

O algoritmo de busca exaustiva, foi desenvolvido de maneira a pesquisar todos os possíveis padrões de corte. A ideia para geração dos padrões de corte se baseou na premissa de iniciar gerando um padrão somente com as peças de maior tamanho a serem cortadas. Se esse padrão gerar um desperdício maior que alguma peça a ser cortada, ele não é um padrão viável e será descartado. Em seguida retira-se uma das peças e a substitui por uma peça de tamanho imediatamente menor e se faz a verificação da viabilidade ou não do padrão, seguindo essa ideia até que se alcance a última combinação que deverá ser conter somente peças do menor tamanho possível. Buscando explanar melhor a ideia é descrito a seguir, de forma explicativa um algoritmo dividido em 8 passos.

Passo 1: Entrada: comprimento da barra padrão $(c)$, número de peças(i), medida de cada peça medida a ser cortada $(p(i))$.

Passo 2: Verificar o maior número possível $(n p)$ de cada tamanho de peça $p(i)$ a ser cortada em cada barra: número possível da cada peça: $n p(i)=c / p(i)$

Passo 3: Arredondar para baixo o número possível da peça $n p(i)$ quando este resultar em um valor decimal.

Passo 4: Gerar os padrões com laços de repetição com a maior quantidade possível de cada peça a compor um padrão, reduzindo sucessivamente as quantidades e aumentando as quantidades das peças de medida menor.

Passo 5: Armazenar todos os padrões encontrados na matriz "matriz padrão" sendo que cada coluna representa um padrão encontrado. 
Passo 6: calcular o desperdício de cada coluna padrão gerada, considerando todos os valores possíveis encontrados, sejam positivos ou negativos.

Passo 7: executar um teste para cada coluna da "matriz padrão" de forma a validar (ou não) o padrão, eliminando padrões que tenham desperdícios negativos que são valores desconsiderados no problema e armazenar os padrões validados na "matriz padrão válido".

Passo 8: executar a saída de dados, em um arquivo tipo ".txt", onde os padrões devem estar dispostos em uma matriz ( $i \times k)$ onde i é o numero de peças de tamanhos distintos e $k$ é a quantidade de padrões encontrados.

Foram realizados testes para o funcionamento do algoritmo de busca exaustiva, levando em conta inicialmente a produção de peças de 3 diferentes tamanhos. Nesse caso, foi obtida a geração de todos os padrões possíveis dentro de um tempo pequeno (menor que $1 \mathrm{~min}$ ).

Aplicando porém o algoritmo no problema real apresentado nesse artigo, o qual envolve peças de 13 diferentes tamanhos, não conseguiu explorar todo o espaço de busca mesmo após 3 horas de execução. Para execução do algoritmo foi utilizado um computador com processador Intel Core i5 $(2.4 \mathrm{GHz})$ e $8 \mathrm{~Gb}$ de memória RAM.

Mesmo por meio de uma análise simples, é fácil notar que em um problema desse porte há grande número de combinações geradas, sendo assim, nesse caso trata-se de um problema de explosão combinatorial. Maiores detalhes sobre esse o aumento do espaço de busca para esse tipo de problema podem ser vistos em FLORIAN et al, 1980.

Buscou-se ainda obter resultados parciais para o algoritmo de busca exaustiva quando aplicado ao problema em estudo, com peças de 13 medidas distintas. Neste caso, na etapa de entrada de dados foi definida uma quantidade máxima de padrões a serem encontrados, para utilizar esse parâmetro como critério de parada para a execução do algoritmo. Notou-se, porém, que para problemas de maior porte (como o exemplo envolvendo 13 medidas distintas), o algoritmo de busca exaustiva fica concentrado na exploração em profundidade de uma determinada região do espaço de busca não sendo capaz de percorrer todo o espaço. Os padrões resultantes da busca exaustiva para 13 medidas após $3 \mathrm{~h}$ de execução, foram formados pela combinação de medidas das 5 primeiras peças somente.

\section{Algoritmo de Busca Aleatória}

Buscando a explorar o espaço de busca de uma forma mais ampla, mesmo que não sejam identificados todos os padrões de corte existentes, foi proposto um segundo algoritmo estruturado (BORATTI e OLIVEIRA, 2013), chamado de algoritmo de busca aleatória.

A ideia principal é evitar uma busca exaustiva em profundidade (CUNHA, 1999) em uma só região do espaço de busca, mas sim, verificar aleatoriamente possíveis padrões de corte, formados por diferentes medidas sorteadas considerando uma distribuição uniforme de probabilidade de ocorrência de cada medida. 
Aproveitando a ideia, foram ainda adicionadas algumas melhorias como a inclusão do desperdício ocasionado pela largura utilizada pela serra no processo de corte e também da quantidade máxima de peças a serem cortadas em um padrão, visto que essa última pode ser uma restrição de máquina, pois á de se considerar que algumas máquinas que executam cortes de forma automática podem ter um limite de quantidade de cortes a serem executados na mesma peça, o que poderia tornar um padrão, embora matematicamente aceitável, em um padrão inviável para uso industrial.

A seguir, para auxiliar o entendimento do funcionamento do algoritmo é mostrado seu funcionamento utilizando 7 passos:

PASSO 1: Entrada: comprimento da barra padrão (c), comprimento de cada medida a ser cortada $p(i)$, quantidades, largura da serra, máximo de peças distintas a serem cortadas em um padrão, quantidade esperada de padrões válidos.

PASSO 2: Verificar o maior número $n(i)$ possível da peça $p(i)$ a ser cortada em cada barra: maior número possível da peça $p(i)=c / p(i)$

PASSO 3: Arredondamento: $n(i)=$ Arredondar para baixo (maior número possível da peça $p(i)$ ). Guardar esses resultados no vetor "QtdeMáx" que representa a quantidade máxima possível de ser cortada da peça $p(i)$ em uma barra padrão.

PASSO 4: Geração dos padrões:

4.1: Gerar um vetor binário (exemplo): $\left[\begin{array}{llllll}1 & 0 & 0 & 1 & 0 & \ldots\end{array}\right]$ onde "1" significa fazer e " 0 " significa não fazer, a peça $p(i)$.

4.2: Gerar um vetor aleatório no intervalo $[0,1]$ da mesma dimensão do vetor gerado no passo 4.1, por exemplo: $\left[\begin{array}{llllll}0,76 & 0,09 & 0,85 & 0,5 & 0,08 & \ldots\end{array}\right]$

4.3: Multiplicar elemento a elemento o vetor do passo 4.1 pelo do passo 4.2 , obtendo um vetor que significa produzir ou não um certo percentual da peça $p(i)$. Neste exemplo tem-se como resultado: $\left[\begin{array}{lllll}0,76 & 0 & 0 & 0,5 & 0\end{array} \ldots\right]$

4.4: Multiplicar o vetor obtido no passo 4.3 pelo vetor de quantidades máximas (QtdeMáx) do passo 2. Assim é obtido o percentual da quantidade máxima a ser cortado em um padrão de corte. Neste exemplo, seria cortado $76 \%$ da quantidade máxima da peça 1 e $50 \%$ da quantidade máxima da peça 4.

4.5: Arredondar cada elemento do vetor para o inteiro mais próximo, obtendo assim um padrão de corte.

PASSO 5: Validação do padrão de corte obtido:

5.1: Multiplicar cada quantidade obtida pelo comprimento de cada peça obtendo o comprimento total a ser cortado. Por exemplo:

$\begin{array}{lllllll}\text { Padrão gerado: } \quad 5 & 0 & 0 & 1 & 0\end{array}$

Medidas das peças: $50 \quad 60 \quad 100 \quad 200 \quad 210$

Nesse exemplo: têm-se:

$(5 \times 50)+(0 \times 60)+(0 \times 100)+(1 \times 200)+(0 \times 210)=450$

5.2: Calcular o desperdício de cada coluna padrão gerada 
$($ Desperdício do padrão gerado $)=($ Comprimento da barra $)-($ Comprimento calculado no passo 5)

Nesse caso, se a barra padrão medisse $300 \mathrm{~cm}$ por exemplo, seria obtido:

(Desperdício do padrão gerado) $=300-450$

(Desperdício do padrão gerado $)=-150$

5.3: Teste de validação do padrão de corte

Executar o teste:

Se [(Desperdício do padrão gerado $) \leq 0]$

E [(Desperdício do padrão gerado $) \leq$

(número máximo de peças a serem cortadas em um padrão)]

Então:

Aproveita-se o padrão, ou seja, esse padrão será válido e será guardado na coluna $i$ da matriz: Padrão Válido $(i \times j)$

Caso contrário:

Volta ao passo 4.

PASSO 6: Critério de parada: obtenção de uma quantidade pré-definida pelo usuário, de padrões válidos.

PASSO 7: Saída de dados: Matriz Padrão Válido ( $i$ × j).

No trabalho aqui apresentado, essa saída foi gravada pelo algoritmo em um arquivo de texto (.txt), o qual foi depois importado para a planilha de cálculo, de modo a alimentar as células que contém os padrões para que dessa forma se possa ter os parâmetros necessários para executar o Solver (Excel).

O algoritmo foi programado utilizando o software Scilab, o qual é um programa de código aberto voltado à computação numérica e, portanto, não possui custo de licença. $O$ download gratuito desse software pode ser obtido em www.scilab.org.

$\mathrm{Na}$ aplicação apresentada nesse artigo, na entrada de dados foram informadas as quantidades e comprimentos mostradas na Tabela 1. Foi ainda neste caso específico, considerado como 5 peças, o número máximo de peças diferentes que pode existir em cada padrão. Essa última informação é importante de forma a buscar simplificar o processo de corte não induzindo o operador da máquina a muitos cortes de tamanho diferentes, caso o processo não for totalmente automatizado. Essa informação pode ser desconsiderada no algoritmo, se for colocado como número máximo de peças diferentes que pode existir em cada padrão, o próprio número de diferentes peças a serem executadas.

Também ainda nesse caso, foi considerada como quantidade máxima de padrões válidos, admitindo repetições, o valor de 230 padrões. Considerou-se 230 padrões, pois se adicionou um coeficiente de segurança 30 padrões que poderiam repetir (esse valor foi aleatório pois não há como saber o número de padrões repetidos que podem ser gerados), para se chegar no mínimo de 200 padrões não repetidos. 
Em seguida o Algoritmo de busca aleatória gerou 230 padrões possíveis, dentro dos parâmetros indicados na entrada de dados. Estes padrões possíveis poderiam ainda admitir repetições, ou seja, poderiam existir padrões de corte idênticos.

O próximo passo do algoritmo consistiu em encontrar os valores padrões válidos sem repetições (esse procedimento é feito pelo próprio algoritmo), no qual foram encontrados 220 padrões do universo de 230 padrões encontrados anteriormente. A Figura 3 mostra uma parte destes padrões não repetidos.

Após execução do algoritmo, foram escolhidos aleatoriamente 200 padrões de corte diferentes. Essa quantidade de padrões foi definida em 200 levando em conta a limitações de software, pois o a ferramenta Solver do Microsoft Excel, suporta problemas contendo até 200 variáveis inteiras, neste caso, 200 padrões de corte. Essa informação está disponível em: https://support.office.com/ptbr/article/Especifica\%C3\%A7\%C3\%B5es-e-limites-do-Microsoft-Excel-1672b34d7043-467e-8e27-269d656771c3).

\section{MODELO DE OTIMIZAÇ̃̃O}

Para resolução do problema foi formulado um modelo de programação linear (ARENALES et al., 2015), o qual teve por objetivo a minimização do desperdício de matéria prima no processo de corte, que nesse caso é formado pela soma das sobras de eletrodutos (ponteiras das peças) com o desperdício gerado pela largura da serra cortante em cada corte efetuado.

Os padrões de corte foram gerados com auxílio do algoritmo aleatório de geração de padrões apresentado nesse artigo, por meio do qual foi obtida uma matriz de padrões a qual foi utilizada para alimentar o modelo de otimização. Como saída do modelo, após a resolução, foram obtidas as quantidades a serem executadas de cada padrão de corte de modo a atender às demandas estipuladas de cada medida com o menor desperdício possível. Foi ainda considerada na formulação do modelo matemático, a possível existência de estoques, o que pode facilitar $\mathrm{o}$ atendimento a pedidos extras.

O modelo matemático é apresentado a seguir.

Parâmetros:

$n$ : número de padrões de corte pré-definido pelo usuário;

$D_{i}$ : Desperdício de matéria prima no padrão de corte $i(i=1, \ldots, n)$;

$E_{k i}$ : Quantidade a ser cortada do eletroduto $k$ no padrão $i$

$Q_{\min (k)}$ : Demanda ou quantidade mínima a ser produzida do eletroduto $k$.

$\mathrm{Q}_{\text {máx(k) }}$ : Demanda ou quantidade máxima a ser produzida do eletroduto $k$.

Variáveis de decisão:

Pi: Quantidade a ser produzida do padrão de corte $i(i=1, \ldots, n)$.

Modelo:

Minimizar $D_{1} P_{1}+D_{2} P_{2}+D_{3} P_{3}+\ldots+D_{n} P_{n}$

Sujeito às restrições:

Demanda_Min_Eletroduto_1) $E_{11} P_{1}+E_{12} P_{2}+E_{13} P_{3}+\ldots+E_{1 \mathrm{n}} P_{n} \geq Q_{\min (1)}$

Demanda_Máx_Eletroduto_1) $E_{11} P_{1}+E_{12} P_{2}+E_{13} P_{3}+\ldots+E_{1 n} P_{n} \leq Q_{\text {máx(1) }}$

Demanda_Min_Eletroduto_2) $E_{21} P_{1}+E_{22} P_{2}+E_{23} P_{3}+\ldots+E_{2 n} P_{n} \geq Q_{\min (2)}$

Demanda_Máx_Eletroduto_2) $E_{21} P_{1}+E_{22} P_{2}+E_{23} P_{3}+\ldots+E_{2 n} P_{n} \leq Q_{\text {máx(2) }}$ 


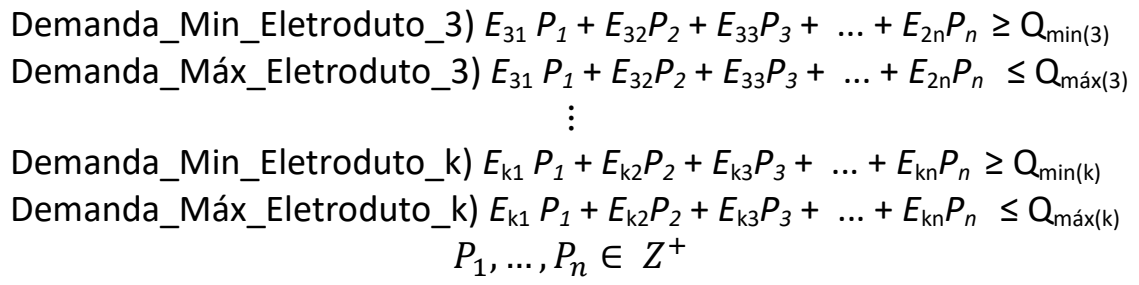

\section{PLANILHA PARA RESOLUÇÃO - FERRAMENTA SOLVER}

Após obtidos os padrões de cortes com auxílio do algoritmo de busca aleatória desenvolvido nesta pesquisa, os padrões gerados foram então transferidos para uma planilha de cálculo a qual é parcialmente mostrada na Figura 3.

Figura 3 - Padrões de corte.

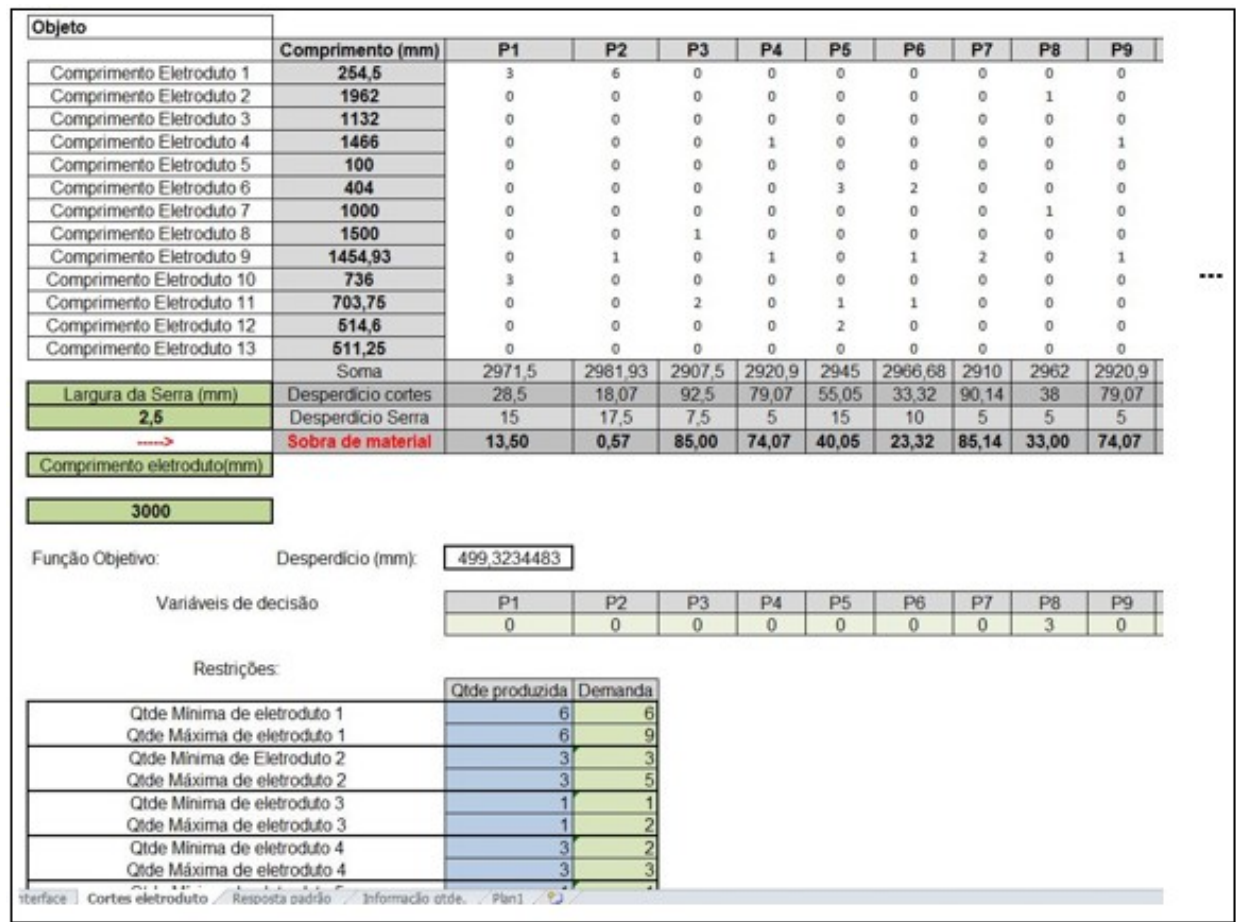

Fonte: Autoria própria (2020).

Para resolução do modelo matemático, optou-se pela ferramenta Solver (a qual é um suplemento da planilha Microsoft Excel), utilizando o método LP Simplex conforme mostrado na Figura 4. 
Figura 4 - Parâmetros do Solver.

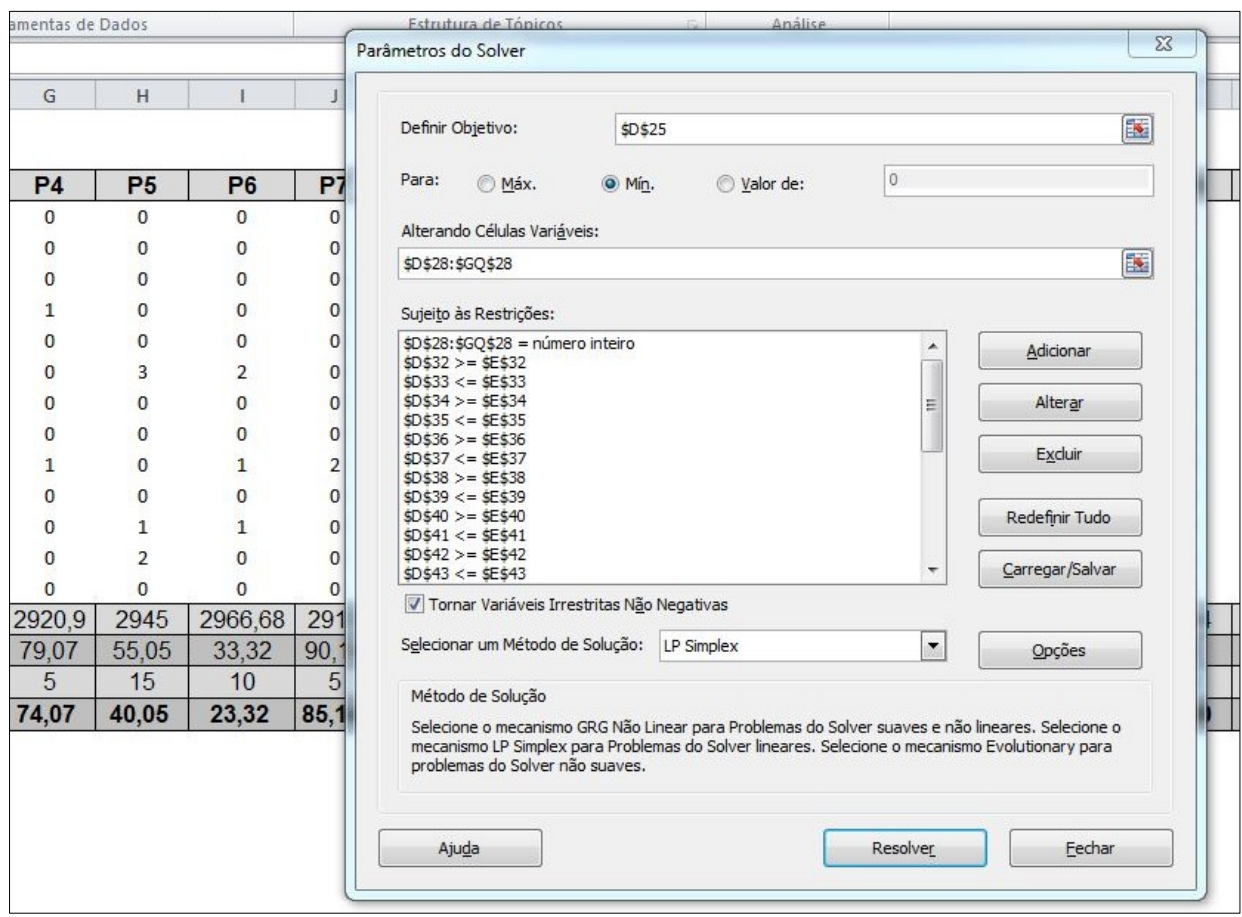

Fonte: Autoria própria (2020).

\section{RESULTADOS}

Neste trabalho foi abordada uma aplicação industrial que envolve o corte de peças de forma linear, o qual é chamado de corte unidimensional. Para estudar o problema foi abordado um problema prático que neste caso foi o processo de corte de eletrodutos. Este tipo de problema envolvendo cortes, é bastante estudado na literatura já possuindo uma formulação matemática de programação linear definida. Porém, um processo dificultoso para esse tipo de problema é a obtenção dos padrões de corte, pois pode existir um elevado número de possibilidades.

Sendo assim, buscou-se através de pesquisas, estudar um método de otimização que pudesse ser de fácil implementação principalmente em pequenas empresas, trazendo possíveis melhorias no processo produtivo.

Após desenvolvido em implantado o método numa empresa piloto, foi obtido redução no desperdício de matéria prima de aproximadamente $5 \%$, se comparado aos métodos empíricos utilizados no cotidiano.

Pode ser uma estratégia interessante na empresa, limitar da quantidade de padrões que serão obtidos como resposta a partir da execução do modelo matemático de modo a evitar um grande número de programações diferentes da máquina de corte, pois essas programações muitas vezes envolvem custos bem como tempo para operação, o que pode influenciar no custo final do produto.

Vale salientar que um importante objetivo desse trabalho foi demonstrar que é possível implementar um modelo científico para auxílio à decisão no processo de cortes com baixo custo pois a metodologia apresentada utiliza um software de programação de uso livre (Scilab) em combinação com a planilha 
Microsoft Excel, a qual geralmente já é utilizada nas empresas de forma que não há necessidade da compra de novas licenças podendo assim conseguir melhorias significativas quanto ao desperdício, contribuindo com questões ambientais e em consequência disso, reduzindo custos produtivos.

Embora esta aplicação tenha sido a motivação para o trabalho, procurou-se desenvolver uma metodologia genérica. Desta forma, o algoritmo proposto pode ser utilizado em aplicações diversas envolvendo cortes unidimensionais como por exemplo no corte de barras, bobinas, tubos, entre outro podendo ser uma referência para outras aplicações.

Quanto aos dois algoritmos desenvolvidos, indica-se a utilização de ambos, conforme a dimensão do problema a ser resolvido. Considerando problemas de pequeno porte, o algoritmo de busca ordenada permite encontrar todos os padrões possíveis, sendo que quando estes são utilizados para alimentar a planilha de resolução obtém-se a solução ótima do problema. Entende-se por problemas de pequeno porte, aqueles que possuem poucos tipos de peças distintas (classificamos como pequeno porte para a metodologia aqui apresentada como até 6 tipos diferentes de peças) e ainda peças de tamanho grande de forma a não haver muitas peças cortadas de uma só peça padrão, buscando evitar a grande expansão no número de diferentes combinações.

Aplicando porém, o algoritmo de busca ordenada ao problema real apresentado nesse artigo, não foi possível explorar todas as combinação mesmo após $15 \mathrm{~h}$ de execução, observando-se ainda que algumas das medidas foram sequer utilizadas nos padrões, o que inviabilizou o planejamento de cortes.

Quando considerado o segundo algoritmo (algoritmo de busca aleatória), este mostrou apresentar resultados em tempo significativamente menor quando comparado ao primeiro (algoritmo de busca ordenada). Este segundo algoritmo é indicado para problemas de grande porte, pois permite percorrer aleatoriamente todo o espaço de busca, havendo nesse caso um conjunto de padrões que envolve todas as medidas a serem cortadas, porém, há o inconveniente do algoritmo de busca aleatória não gerar todos os padrões de corte possíveis, o que faz com que o modelo gerado por meio da alimentação da planilha com os padrões obtidos resulte numa solução não ótima.

Em relação ao desperdício de matéria prima na empresa utilizada como objeto de estudo houve redução de aproximadamente $1,7 \%$ se comparado os resultados obtidos pelo modelo matemático utilizando o algoritmo de busca aleatória, com o procedimento adotado na empresa, que utiliza um planejamento empírico, sem uso de qualquer modelo de otimização. Foram nesse caso resolvidos problemas para vários cenários (diferentes demandas) onde para todos os cenários houve redução de desperdícios sendo que em um dos cenários foi obtido aproximadamente $5 \%$ de redução.

Para obtenção dos padrões foi utilizado como critério de parada a delimitação de tempo de execução do algoritmo de busca aleatória, o qual foi fixado em 15 minutos para todos os cenários. Uma sugestão para pesquisas futuras, seria verificar se como o aumento do tempo de execução do algoritmo exerce influência na redução do desperdício. 
Outra sugestão para futuros trabalhos é a aplicação de heurísticas que possam explorar o espaço de busca de forma a encontrar padrões de corte, para que estes sejam utilizados no modelo de otimização.

\section{CONCLUSÕES}

Em relação à resolução do modelo gerado, pode ser interessante para a empresa a limitação da quantidade de diferentes padrões de corte a serem obtidos como resposta do após a execução do modelo matemático. Essa estratégia auxilia a prática na empresa quando o ato de preparação da máquina de corte corre de forma manual, possui altos custos, ou ainda demanda um tempo elevado pois evita muitas programações diferentes para a máquina que executa cortes. Essa estratégia não foi utilizada na resolução do problema estudado nesse trabalho.

Uma limitação desse trabalho foi a quantidade de diferentes padrões de corte a serem utilizados no modelo matemático, o qual, devido à utilização do Microsoft Excel, não pôde exceder a 200 padrões, pois este é a quantidade máxima de variáveis inteiras que o Solver do Excel admite. Nesse caso, como sugestão pode-se utilizar solvers, porém, de modo geral estes possuem a desvantagem referente ao custo das licenças de utilização quando disponibilizados ao ambiente industrial (alguns solvers possuem licenças acadêmicas gratuitas, como por exemplo o Cplex).

Outras sugestões ainda para trabalhos futuros poderão ser verificadas na implementação prática no ambiente industrial, como por exemplo, a criação de uma interface para o sistema, a qual simplifique sua utilização o que indica um caminho promissor para novos trabalhos. 


\title{
Generating patterns for the one- dimensional cut problem: an Industrial application
}

\begin{abstract}
This work presents a study on a one-dimensional cut problem from which an algorithm was developed to aid in the generation of cut patterns. Based on the patterns generated with the algorithm and after verifications in works in the literature, an optimization model was proposed involving a linear programming problem whose resolution was implemented with the aid of the Solver supplement, available in Microsoft Excel software. The algorithm developed to generate the standards was implemented in Scilab language, as it is a free license software. The optimization model was inspired by an existing problem in an industry that works with conduit cutting, but care has been taken to generalize it so that it can be used in other applications involving one-dimensional cuts. Results showed that the model developed is a good tool to aid decision making that opens the possibility for the search for waste reduction involving raw material, indicating a promising area for computational development and future research.
\end{abstract}

KEYWORDS: Algorithm. Cuts problem. Optimization. 


\section{REFERÊNCIAS}

AGUILAR, Luis Joyanes. Fundamentos de programação: Algoritmos, Estruturas de dados e objetos. São Paulo: Mcgraw-hill, 2008. 690 p.

ANDRADE, E. L., Introdução à Pesquisa Operacional: Métodos e Modelos Para a Análise De Decisão. 2. ed. Rio de Janeiro: LTC, 2000.

ARENALES, M. et al. Pesquisa Operacional. 2ae edição. Rio de Janeiro: Elsevier, 2015.

BORATTI, I. C.; OLIVEIRA, A. B. Introdução à Programação Algoritmos. 4. ed. Florianópolis: Visual Books, 2013.

BORGES NETO, M. R.; CARVALHO, P. Geração de energia elétrica: Fundamentos. São Paulo: Érica Ltda., 2013.

BUENO, Fabrício. Otimização gerencial com EXCEL. Florianópolis: Visual Books, 2007. 136 p.

CUNHA, R.R. M.; DIAS, A. Algoritmo de otimização de corte unidimensional análise de dados, Anais, XV Congresso brasileiro de engenharia mecânica, 10 p., 1999.

FLORIAN, M.; LENSTRA, J. K.; KAN, A. H. G. R. Deterministic production planning: Algorithms and complexity. Management Science, v. 26, n. 7, p. 669-679, 1980. crossref

FORBELLONE, André Luiz Villar; EBERSPÄCHER, Henri Frederico. Lógica de programação: A construção de algoritmos e estruturas de dados. 3. ed. São Paulo: Prentice Hall, 2005. 218 p.

GOLDBARG, M. C.; LUNA, H. C. L. Otimização Combinatória e Programação Linear: Modelos e Algoritmos. 2a‥ ed. Rio de Janeiro: Elsevier, 2005.

LOESCH, Cláudio; HEIN, Nelson. Pesquisa Operacional: Fundamentos e modelos. São Paulo: Saraiva, 2009.

MOURA, C.R. et al. Otimização de cortes bidimensionais em chapas através da aplicação de modelos matemáticos. v. 15, n. 3, p. 59-75, Jul./Set. 2019. crossref 
OLIVEIRA, B.; SALVADOR, R.; SILVA, V. M. D. Otimização do Problema de Corte Unidimensional: Um Estudo de Caso na Indústria Papeleira. Anais: EEPA, Encontro de Engenharia de Produção Agroindustrial, 7, Campo Mourão- PR, 2013. crossref

SILVEIRA JÚNIOR, J. A.; PINHEIRO, P. R.; THOMAZ, A. C. F. Otimização das Perdas em Cortes Guilhotinados para Bobinas de Aço na Indústria Metalmecânica, Anais, SBPO: Simpósio Brasileiro de Pesquisa Operacional, 36 p. 1759 - 1770, 2004.

SIMONE, G. A. Centrais e aproveitamentos hidrelétricos: Uma introdução ao estudo. São Paulo: Érica, 2000.

WALENIA, P. S. Projetos Elétricos Industriais. Curitiba: Base Editorial, 2010.

WAVRZYNCZAK, H. C.; ULBRICHT, G.; TEIXEIRA, E. S. M. Modelo Matemático para Cortes de Barras de Aço no Processo de Fabricação de Triângulos. HOLOS, [S.I.], v. 8, p. 252-261, jan. 2016. Disponível em:

<http://www2.ifrn.edu.br/ojs/index.php/HOLOS/article/view/3605/1326>. Acesso em: 16 out. 2019.

Recebido: 03 Set. 2020

Aprovado: 14 Out. 2020

DOI: 10.3895/gi.v16n4.13118

Como citar:

ULBRICHT, G. et al. Algoritmos para geração de padrões aplicado ao problema de corte unidimensional. $\mathbf{R}$.

Gest. Industr., Ponta Grossa, v. 16, n. 4, p. 272-287, Out./Dez. 2020. Disponível em:

https://periodicos.utfpr.edu.br/revistagi

Correspondência:

Gerson Ulbricht

Rua dos Imigrantes, 445, Bairro Rau, Jaraguá do Sul, SC, Brasil.

Direito autoral: Este artigo está licenciado sob os termos da Licença Creative Commons-Atribuição 4.0

Internacional. 